\title{
2019 Women in Sport and Exercise Conference Abstracts
}

\section{Abstract Summaries \\ Open Communications}

Gender Gap in Painful Knees: PFJ \& ACL - 'Odd Couple' Biomechanics and Neuromuscular Differences Between Females and Males

Mimi Zumwalt ${ }^{1}$

${ }^{I}$ Texas Tech University Health Sciences Centre, TX, USA

Since 1972, after Title IX was passed governing gender equality in scholastic athletics, an exponential rise in musculoskeletal trauma accompanied the explosion of female sports participation; mostly regarding knee injuries which comprise up to $50 \%$ of all sporting activities. Specifically, the incidence of patellofemoral $(\mathrm{PF})$ pain $(25 \% />2 \mathrm{x})$ and anterior cruciate ligament (ACL) injuries (2-8x) occurs more often in females compared to males. Interestingly, female knee (ACL) injuries occurring during sports participation parallels the onset of puberty and release of the sex-specific hormone, oestrogen. The ramifications of ACL tears put a tremendous strain on the injured athlete and society, not only physically, but also psychologically and economically i.e. disability/financial cost. Various theories have attempted to explain this gender disparity in knee injury incidence. Both intrinsic and extrinsic factors play a role including lower extremity alignment, landing biomechanics and differential neuromuscular activation; each is implicated in plausible trauma causation. Inherent morphology of bones/joints above and below the knee can predispose female athletes to PF injuries and/or ACL tears. Since anatomical variation cannot be altered, current research aims to find alternative ways to minimise painful symptoms/injury risk. Studies indicate that strength and conditioning programs which incorporate plyometrics into the training regimen have demonstrated success in reducing/preventing knee trauma by as much as $50 \%$. By targeting specific lower extremity muscle groups in female athletes, and encouraging athletes to repeatedly practise how to land from a jump, reflexive mechanism will hopefully occur within the body, i.e. posture control during sports manoeuvres in order to protect them from incurring knee trauma.

Increased Injury Risk in Short, Middle and Long-Distance Female Runners

Anna Kosciuk ${ }^{1,2}$ and Nicola Swann ${ }^{1}$

${ }^{1}$ Kingston University, UK;

${ }^{2}$ Profeet Sports Lab, London, UK

Running-related injuries constitute a major health concern, especially with increases in recreational running and a growing number of distance running events. This study aimed to investigate the effects of gender and weekly distance on lower extremity injury prevalence in runners. A retrospective cohort study was designed to investigate the prevalence of running-related injuries and establish trends in short-, middle- and longdistance runners. Anonymised self-reported data relating to 386 females and 614 males were sampled and analysed. Both injured and non-injured individuals were included. Runners in all distance population groups were found to have a high risk of running-related traumas (80.5 - 86.9\%), but females reported greater injury rates than males in each distance group, with the greatest difference observed in middle-distance runners (females $91.5 \%$, males $76.0 \%$ ). Knee injuries were the most common traumas reported, followed by the foot and shank. A significantly higher number of hip injuries were reported by females than males. Overall, females were found to be at a high, and significantly greater, risk of running-related issues compared to males, which was not explained by training habits. Although males and females showed similarity in patterns of injury, the underlying pathway of those injuries might differ between runners due to various internal factors associated with gender. Further research should focus on investigating how foot and lower limb mobility, body anatomy, as well as hormonal status affects movement biomechanics in females to develop strategies to protect females from runningrelated issues through exercise, footwear recommendation and gait education.

\section{USA Women's Rugby-7s Non-Contact Injury Incidence}

Victor Lopez, $\mathrm{Jr}^{1-3}$, Batool Quteishat ${ }^{1,4}$, Richard $\mathrm{Ma}^{1,3,5}$, Christian Victoria ${ }^{1,6}$, Meryle G Weinstein ${ }^{1,6}$, Patria A Hume ${ }^{2}$, Samuel Y Haleem ${ }^{1,7}$, Fady Gries ${ }^{1,8}$, and Answorth A Allen ${ }^{1,9-11}$

${ }^{I}$ Rugby Research and Injury Prevention Group, NY, USA;

${ }^{2}$ Auckland University of Technology, Sports Performance Research Institute, New Zealand;

${ }^{3}$ Northeast Rugby Academy, USA Rugby \& USOC-Community Olympic Development Program, NY, USA;

${ }^{4}$ Loughborough University, UK;

${ }^{5}$ University of Missouri, MO, USA;

${ }^{6}$ New York University, NY, USA;

${ }^{7}$ City University of New York, NY, USA;

${ }^{8}$ West Virginia School of Osteopathic Medicine, WV, USA;

${ }^{9}$ Sports Medicine and Shoulder Service, Hospital for Special Surgery, NY, USA;

${ }^{10}$ National Basketball Association, New York Knickerbockers, NY, USA;

${ }^{11}$ USA Basketball, CO, USA

Despite tremendous growth of female participation in Rugby-7s, there are limited data on injury epidemiology of women's Rugby-7s. The aim of this study was to prospectively report non-contact injury incidence and causes among US women's under-19 to elite Rugby-7s' players over 2010-2015 using the Rugby Injury Survey \& Evaluation (RISERugby) methodology. Non-contact causes of injury occurred frequently $(26.5 / 1000 \mathrm{ph} ; 29 \%$ of all injuries; $n=167$ ) over the study period (classic-non-contact $(\mathrm{CNC})=92 \%$, $n=153$; other-non-contact $(\mathrm{ONC})=8 \%, n=14 ; \mathrm{p}<0.001)$. Overall, noncontact injury incidence (time-loss and medical attention) were found at similar rates among backs $(58 \%, 23.9 / 1000 \mathrm{ph})$ versus forwards $(42 \%$, 19.3/1000 ph; RR:1.04, $p=0.816$ ). CNC time-loss injuries were observed 
at similar rates among positions (backs $6.1 / 1000 \mathrm{ph}$; forwards 5.9/1000ph, RR:1.03, $p=0.933)$. Non-contact injuries resulted in 58.4 mean days absent (CNC 58d; ONC 60d). The study's findings of non-contact injury associated with the open play phase (39\%) and periods of intense running/ sprints support the need for training and conditioning necessary to meet Rugby-7s' demands for women. The high muscle and tendon injury rates $(31 \%)$ could be reduced with preseason and in-season conditioning. The high rates of joint and ligament injuries (39\%), particularly among US females' knees and ankles, could be reduced by increasing muscular load tolerance and proprioception during training and by including open field manoeuvres. The high recurrent injury rates at $40 \%$ may be reduced with proper education on the importance of appropriate post-tournament medical care and return-to play programmes.

\section{"We're Here to Become Good People, Not Good Footballers!": Uncovering Meaning Through an Ethnographic Exploration of Girls' Experiences in a Recreational and Restorative Football Programme in Central Scotland}

Chelsea Raymond ${ }^{1}$

${ }^{1}$ University of Stirling, $U K$

In light of the historic qualification of Scotland's Women National Team for their first World Cup finals, football participation of women and girls in Scotland has grown tremendously. Reports abound with statistical insights into reasons for motivation and participatory trends; however, what lacks is a thorough understanding of the intermediate outcomes of participation, especially in recreational environments. This ethnographic study explored the lived experience of a group of at-risk girls participating in a girls'-only recreational and aspirational football programme in Scotland. With the primary aims of life-skill development, increasing school retention and promotion of physical activity in this historically marginalised population, this programme utilises football as a vehicle for change. Wholly focused on 'becoming good people, not good footballers', this programme offers these young girls a safe space to learn and grow through football. Investigatory aims focused specifically on the meanings associated with relationships, identity and gender within the context of recreational football. This paper presents early findings from data collected through fieldwork observations, informal and formal interviews, and participatory arts-based focus groups. Despite the lack of formal games and competition, the importance of 'team' identity proved to significantly impact the development of individual identity. Another significant component of their experience was the importance of the developed culture. Their lived experience, sense of identity and culture are conceptualised through the theoretical lens of the ethic of care. These insights aim to contribute to the budding literature on the creation of caring sporting environments.

\section{Zumba Fitness Improves Aerobic Fitness and Psychological Wellbeing in Healthy Women}

Anne Delextrat ${ }^{1}$, Sarah Warner ${ }^{1}$, Sarah Graham ${ }^{1}$, and Emma Neupert ${ }^{2}$

${ }^{I}$ Oxford Brookes University, UK;

${ }^{2}$ Winchester University, $U K$

The aim of this study was to investigate the effects of an eight-week exercise intervention based on Zumba fitness classes on physiological and psychological outcomes in healthy women. Maximal oxygen consumption (VO2max), body composition, physical self-perception and psychological wellbeing were assessed before and immediately after eight weeks of Zumba performed three times weekly (Zumba group, $n=22$, age: $M=26.6$, $S D=5.4$ years old; height: $M=165.8, S D=7.1 \mathrm{~cm}$ ) or no intervention (control group, $n=22$, age: $M=27.9, S D=6.0$ years old; height: $M=164.7$, $S D=6.2 \mathrm{~cm}$ ). All variables were analysed by a two-way (Group x Time) analysis of variance with repeated measures, and a Bonferroni post-hoc test. Pearson correlation coefficient assessed the relationship between changes in anthropometric, physiological and psychological variables. Zumba provided significant positive changes in $\mathrm{VO}_{2} \max (+3.6 \%)$, self-perception of physical strength $(+16.3 \%)$ and muscular development $(+18.6 \%)$, greater autonomy $(+8.0 \%)$, and purpose in life $(+4.4 \%)$. No significant changes were observed in the control group. In addition, some psychological changes were significantly correlated to body fat at baseline and changes in fitness. These results are in accordance with those from the literature for aerobic fitness and body fat and highlight that Zumba is beneficial to improve fitness and wellbeing in healthy women but might not be appropriate on its own for weight loss.

Weight on the Bar vs. Weight on the Scale: Disordered Eating Pathology in Competitive Female Powerlifters

Maria Luisa Vargas ${ }^{1}$ and Stacy Winter ${ }^{1}$

${ }^{1}$ St Mary's University, UK

Disordered eating research in sport psychology has arisen due to the need to investigate sub-clinical populations. The manifestation of sub-clinical symptoms presents risk to full eating-disorder diagnosis. Researchers have identified the risk of disordered eating development to be heightened in female athletes and those in lean, weight-preoccupation sports. The aim of the study was therefore to explore disordered eating pathology through subjective enquiry in competitive female powerlifters. Methods: Semistructured interviews were conducted with 17 British female powerlifters, competing at national or international level. Three major themes which contributed to disordered eating were identified through inductive thematic analysis; unique sport-specific factors, preoccupation with food, and managing body perceptions. Themes highlighted the conflict between the aim of powerlifting in terms of adding 'weight on the bar', and the weight-class aspect of the sport requiring athletes to monitor 'weight on the scale'. Through their experiences in the sport, a combination of both positive and negative accounts were provided by participants. The unique characteristics of powerlifting, requiring maximal loads to be lifted, created feelings of empowerment by the female athletes. Furthermore, the sport emphasises characteristics traditionally deemed masculine, which defy the norms of femininity. In order to reap the positive benefits, and to alleviate disordered eating development and maintenance, a multi-disciplinary approach is advocated. Discussions amongst athletes and support staff may prove useful in creating an awareness of this mental health concern and ultimately eradicate the negative connotations associated with this 'taboo' topic.

\section{Gender Equity in Sport Governance: Next Steps for Creating Change} Lucy Piggott ${ }^{1}$

\section{${ }^{1}$ University of Chichester, $U K$}

Sport governance remains gender-imbalanced at all levels of leadership across the world, and English sport governance provides no exception. Scholars, activists and practitioners have identified the need to address gender inequity within sport governance. This presentation aims to: (1) Present an overview of key findings from a doctoral study that explored the workings of gender power relations within the governance of two English NGBs: England Golf and the Lawn Tennis Association; and (2) Provide recommendations of how academic research and applied practice can combine to create organisational change towards achieving gender-equitable sport governance. A multi-method approach was used, which consisted of semi structured interviews (primarily with senior leaders and middle managers), participant observation (of general organisational life and specific events) and an analysis of supporting documents (including annual reports, rules and board minutes). There was evidence of both the conservation and resistance of gender power relations that profit 
men across the governance of the two English NGBs. Conservation strategies included gendered rules and structures, gendered recruitment processes, gendered organisational cultures, and greater opportunities for men to accumulate, convert and maintain power. Resistance strategies included changing governance rules, positive action towards women leaders, addressing overtly gendered cultural practices, and providing development opportunities for women leaders. Power continues to operate along gendered lines to reproduce male-dominance within sport leadership numerically, structurally, culturally and agentically. To inform organisational change towards gender equity, academics need to link their recommendations to espoused organisational values and performance to provide motivation for organisations to make genuine, sustainable change.

\section{Educating Generation Z About Gender Equality in Sport: A Social Learning Perspective}

Inge Derom ${ }^{1}$, Hebe Schaillée ${ }^{1}$, Solveig Straume ${ }^{2}$, Helmien Rambaldo ${ }^{3}$, Gill Renfree ${ }^{4}$ and Vanessa Jones ${ }^{4}$

\author{
${ }^{1}$ Vrije Universiteit Brussel; \\ ${ }^{2}$ Molde University College; \\ ${ }^{3}$ The Hague University of Applied Sciences; \\ ${ }^{4}$ University of Worcester, $U K$
}

Although research has reported increases in female participation in sports, women and girls are still under-represented in coaching, leadership and decision-making roles, as well as in sports media. The GETZproject ('Gender Equality Toolkit for Generation Z') looks at the issue of gender inequality from the perspective of the next generation and aims to develop, deliver and evaluate an innovative educational resource specifically designed for Generation $\mathrm{Z}$ on the issues relating to gender equality and equal opportunities in sport. The GETZproject is carried out by a consortium of institutions across seven countries and is co-funded by the Erasmus+ Sport programme of the European Union. Generation $\mathrm{Z}$ are individuals born in the mid-1990s to early 2000s, who frequently interact on social media platforms for a significant portion of their socialising and, consequently, social learning. In order to understand the perceptions, attitudes and actions related to gender equality and equal opportunities in sport among Generation $\mathrm{Z}$, undergraduate students from four institutions and countries were recruited and a total of eight focus groups were conducted. The preliminary results of this research demonstrate that undergraduate students reported common perceptions and experiences across the four participating institutions and countries. Furthermore, no major differences in responses between male and female students were found. Respondents discussed the outspoken advantage for males in the sport industry, in terms of governance, media coverage and participatin (recreational and elite). The development of the GETS-toolkit is elaborated upon, with reference to ongoing challenges related to gender inequality.

\section{Pushing the Boundaries for Commercial Viability and Financial Freedom of Sportswomen \\ Emmanuel Mogaji ${ }^{1}$, Foluké Abi Badejo ${ }^{2}$, Simon Charles ${ }^{3}$, and Jacqueline Millisits $^{4}$ \\ ${ }^{1}$ University of Greenwich, $U K$; \\ ${ }^{2}$ Griffith University, Queensland, Australia; \\ ${ }^{3}$ SWBA Project, UK; \\ ${ }^{4}$ University of Connecticut, Connecticut, USA}

Research about women in sports has often focused on exercise, physical training, nutrition and psychology, while the commercial viability of sportswomen is less explored. This research sought to explore sportswomen's effort and challenges as they move beyond the physical side of sports towards commercial viability and financial freedom. Semi-structured interviews with 24 sportswomen across the UK were carried out. Analysis revealed that sportswomen seldom focus on their commercial viability; they do not consider themselves as brands, as they want to focus on their career. For those who have tried to build their commercial viability, they face challenges such as poor media coverage, consumers and brand attitude towards sportswomen, and not having the right management team. This consequent increased financial vulnerability as sportswomen influences their wellbeing, such as having to delay life's major decisions and decreased mental wellbeing. While the physical side of sports is still essential for women, they need to be mindful of their commercial viability, thinking of how they make money to support themselves and have more financial freedom - pushing boundaries to build themselves as a brand, telling their stories and engaging with the audience. This study contributes to a better understanding of the financial wellbeing of sportswomen. It offers practical implications for sportswomen to take additional responsibilities; governing bodies to support sportswomen; investing in a supportive environment that can assist women in balancing gender roles with a sporting career. Talent managers and coaches also need to be more aware and mindful and supportive of their clients' wellbeing.

\section{A Global Survey of Menstruation and Exercise Habits in $\mathbf{1 4 , 1 8 4}$ Exercising Women}

Georgie Bruinvels ${ }^{1,2}$, Esther Goldsmith ${ }^{1,2}$, Katie Morton ${ }^{2,3}$, Nathan Lewis ${ }^{1,2}$, and Charles Pedlar ${ }^{1,2}$

${ }^{1}$ St Mary's University, UK;

${ }^{2}$ Orreco Ltd;

${ }^{3}$ University of Cambridge, $U K$

Approximately three quarters of women cite their menstrual cycle as a barrier to performance. However, the inter-relationships between the menstrual cycle (e.g. characteristics, symptoms), exercise, diet and lifestyle factors are not well understood. Additionally, where dysfunctions or symptoms are present, medical intervention (e.g. painkillers, hormonal contraception) are often the primary treatment option. Historically there has been limited discussion and education in this area. Few data exist to describe the relationship between these factors in the UK and globally. The aims of this work were to: (1) gain a better understanding of menstrual function in exercising women (2) identify prevalence of hormonal contraception use and menstrual dysfunction; and (3) identify any geographical differences in education, communication and participation in exercise during puberty. A large subset of users of an activity and distance tracking application (STRAVA) from the UK, Ireland, USA, Spain, France, Germany and Brazil were invited to complete a 37-part online questionnaire lasting approximately 10 minutes. Results were analysed descriptively, and associations between variables assessed. 14,184 women participated in the survey. Excluding those not of a reproductive age, $3.5 \%$ had amenorrhea, $6.0 \%$ oligomenorrhea, $42.1 \%$ eumenorrhea, $1.1 \%$ polymenorrhea and $48.4 \%$ were using hormonal contraception. $80.5 \%$ of women reported experiencing menstrual cycle-related symptoms every cycle, and $68.7 \%$ have had to alter their training as a result of their menstrual cycle. Only $25 \%$ of those coached have discussed their menstrual cycle with their coach, and only $15.9 \%$ of women have received education in this area.

\section{Female Athletes, Amenorrhea, and Fertility}

Megan Stanley ${ }^{1}$, Nicola J Rinaldi ${ }^{2}$, Nancy Clark, and Jackie Buell ${ }^{1}$

${ }^{1}$ Ohio State University;

${ }^{2}$ Antica Press LLC

Amenorrhea in biologically female athletes is fortunately becoming less of a taboo topic, particularly with recent publications highlighting the 
importance of having one's period, along with recommendations that menstrual cycle status be treated as the fifth vital sign. However, research on longterm impacts of amenorrhea at different ages is sparse. Two major questions include the effects of timing and duration of amenorrhea on bone density and, similarly, effects of amenorrhea on later fertility. When counselling athletes about alterations in eating and exercise habits to attempt to address the underlying causes of amenorrhea, understanding of these long-term impacts by both clinicians and athletes can be helpful. In this session we will share results from a digital questionnaire sent to alumnae athletes at Ohio State University as a pilot for a larger effort. We assessed history of menstrual dysfunction throughout high school, collegiate and post-collegiate athletic careers, across varied ethnic groups and sports, along with self-reported physician diagnosis, treatments offered and fertility history. We included a lab visit for local participants to assess effects on bone density and resting metabolic rate. The questionnaire outcomes provide unique data to better understand the impact of athletic amenorrhea on later fertility. The laboratory data, when combined with data from additional institutions, will provide much needed insight as to the long-term physical impacts.

\section{Age and Sex Differences in Vascular Function: The Role of Oestrogen}

Sophie M Holder ${ }^{1}$, Ellen A Dawson ${ }^{1}$, Andrew Thompson ${ }^{2}$, Daniel J Green ${ }^{3}$, and Dick HJ Thijssen ${ }^{1,4}$

${ }^{1}$ Liverpool John Moores University, UK;

${ }^{2}$ University of Liverpool, $U K$;

${ }^{3}$ The University of Western Australia, Australia;

${ }^{4}$ Radboud University Medical Center, the Netherlands

Women have a lower incidence of cardiovascular disease (CVD) compared to men, partially due to the protective effect of oestrogen. Vascular function, measured by brachial artery flow-mediated dilation (FMD), predicts CVD risk. Age- and sex-specific differences in FMD are well established; however, interpretation is hindered by the lack of reference values. This study aimed to establish reference values for brachial artery FMD in healthy adults, obtained with strict adherence to expert-consensus guidelines for the measurement of FMD. In a retrospective study design, we combined brachial artery FMD data of 891 healthy adults (age 18-84 years; 348 women). Fractional polynomial regression generated age-specific percentile curves and sex-specific predictive equations for FMD. A t-test explored the independent effect of oestrogen on 11 pairs of healthy age-matched pre- and post-menopausal women. The age-related decline in FMD was linear in both sexes; however, FMD declined at a faster rate in women compared with men $(-0.061 \%$ and $-0.032 \%$ per year increase in age, respectively). There was no difference between pre- and postmenopausal women (pre-menopause $M=$ $6.97, S D=3.22 \%$, post-menopause $M=4.95, S D=1.82 \%$; $p=0.096$ ); however, this exploratory analysis may be clinically meaningful in relation to CVD risk. Ageing is associated with a decline in FMD, even in a healthy population. Oestrogen may contribute to the sex differences in the agerelated decline in FMD. The age-related decline may be slowed by regular exercise, and is therefore recommended to combat the age-related decline in vascular function and reduce CVD risk.

Physical Activity Participation During Pregnancy in Previously Sedentary and Highly Active Women

Ciara O'Hagan ${ }^{1}$ and Louise Turner ${ }^{2}$

${ }^{1}$ Institute of Technology Carlow, Ireland;

${ }^{2}$ Sheffield Hallam University, UK

Regular moderate intensity exercise ( $>150 \mathrm{~min} /$ week) is recommended by the Health Services Executive in Ireland to promote maternal health during pregnancy, but more than $75 \%$ of pregnant women do not meet this recommendation. Little is known about women who were engaged in competitive sport training pre-pregnancy. The aim of this study was to explore the physical activity knowledge and participation during pregnancy of women whose pre-pregnancy activity status ranged from sedentary to elite athletes. An online questionnaire was developed to collect data in five domains: knowledge of physical activity guidelines; physical activity, sport and exercise participation before and during the three trimesters of pregnancy; reasons for changes in activity participation; sources of advice and guidance; and perceived risks of physical activity during pregnancy. The questionnaire was distributed using a snowball sampling approach to recruit adult women who had experienced a pregnancy in Ireland during the past five years. Preliminary data analysis suggests that while most women reduced physical activity frequency, intensity and duration across their pregnancy, previously highly active women were more likely to meet pregnancy physical activity recommendations than those who were previously sedentary or had low activity levels. Women across all pre-pregnancy activity categories received some advice from healthcare professionals but also reported high usage of social media and other online sources to guide their activity participation. These findings are important to consider in the context of public dissemination and promotion of pregnancy physical activity recommendations to both sedentary and previously active women in Ireland.

\section{Introducing This Mum Moves: An Educational Campaign to Support Pregnant Women and New Mothers in Enjoying and Benefitting From an Active Lifestyle}

Marlize de Vivo ${ }^{1}$ and Hayley Mills ${ }^{1}$

${ }^{1}$ Canterbury Christ Church University, UK

The Chief Medical Officer's recommend that pregnant women engage in 150 minutes of moderate intensity physical activity (PA) every week and do muscle strengthening activities twice per week. However, it is estimated that only $3-15 \%$ of women meet these guidelines and that activity levels rarely return to pre-pregnancy levels. This Mum Moves (TMM) is a project aiming to support women to be active throughout motherhood by providing health care profressionals with the tools (training and resources) to deliver physical activiry advice and guidance as part of routine care. A Patient and Public Involvement (PPI) approach adopted during the development phase involved: (1) an online survey of healthcare professionals' knowledge and confidence in providing PA advice and guidance $(n=404)$; and (2) seven focus groups involving pregnant women, new mothers, midwives and health visitors $(n=43)$. Key findings include: advice to be received early in pregnancy, if not pre-conceptually, and continued postnatally; information to be delivered by a trusted professional; clear and consistent advice to be available throughout pregnancy and beyond; generic information to be available in print with more specific guidance and signposting provided by professionals; advice to be part of routine conversations; information about local PA opportunities to be current and accessible through a range of mediums; and campaigning to be inclusive and relevant to the local community. These findings form the foundations on which the TMM project is based, the effectiveness of which will be tested in two pilot sites, before upscaling and launching nationally.

Support for Sport: Short- and Long-Term Impact of a Breast Education Intervention on Adolescent Girls' Breast Knowledge and Attitudes to Breasts

Atefeh Omrani ${ }^{1}$, Jenny Smith ${ }^{2}$, Joanna Wakefield-Scurr ${ }^{3}$, and Nicola Brown ${ }^{1}$

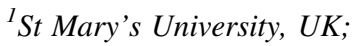

${ }^{2}$ University of Chichester, $U K$;

${ }^{3}$ University of Portsmouth, UK.

Only $12 \%$ of UK 14 -year-old girls meet physical activity guidelines. In our survey study with $>2,000$ girls, $46 \%$ of girls reported that their breasts 
affected their sports participation and $73 \%$ reported $\geq$ one breast-specific concern in sports, with breast bounce being most prevalent. Furthermore, $26 \%$ of girls reported negative feelings about their breasts and $87 \%$ want to learn more about their breasts. This study aims to evaluate the short- and long-term impact of a 50-minute comprehensive breast education intervention on girls' breast knowledge and attitudes towards breasts and breast issues. A mixed method, controlled, longitudinal-cohort-design was employed: two control schools (receiving no intervention) and two intervention schools (receiving the intervention) participated in the study. All four schools completed a validated breast survey pre- and post-intervention, as well as three and six months after the intervention. Additionally, in one intervention school, six focus groups were conducted post-intervention and four months after the intervention. Analysis of survey data established the short- and longterm positive impact of the intervention; participants in the intervention schools $(n=375)$ significantly improved their breast knowledge, and their attitudes towards breasts over a sixmonth period, compared to participants in the control schools $(n=412)$. The focus group findings supported the survey results. Participants described the session as informative and it made them feel less embarrassed about their breasts; they also reported wanting to do more exercise. These findings demonstrate the positive impact of the intervention which may have the potential to impact policy change and inform education guidelines.

\section{Significant Moments and People in Female Coaching Journeys}

Polly Lasota ${ }^{1}$, Luciana De Martin Silva ${ }^{2}$, Thomas Legge ${ }^{3}$, and Ryszard Chadwick ${ }^{3}$

${ }^{1}$ University of Worcester, $U K$;

${ }^{2}$ Hartpury University, $U K$;

${ }^{3}$ Play Rugby USA

Previous research has highlighted the under-representation of women in coaching, focusing primarily on the challenges faced by elite female coaches. A shift in socio-cultural and political landscapes towards equal opportunities for minority groups in society has supported the development of further enquiry into supportive mechanisms for female coaches who have various levels of coaching experience. This is particularly important in contributing to the creation of effective supportive environments that inspire female coaches to pursue and sustain a career in coaching. Hence, the aim of this research was to investigate key moments and people in female coaching journeys that have influenced their personal and professional development (while pursuing and sustaining a career in coaching); moreover, to provide recommendations for future supportive provisions for the development of female coaches. Semi-structured interviews were utilised in order to gain rich insight into female coaching journeys. One initial set of interviews (lasting $\approx 50$ minutes each) included 12 coaches (Level 2 to Level 4 UKCC qualified/or equivalent) from a range of sport specialisms, with a minimum of three years' coaching experience. A second set of interviews was conducted with four of the original coaches three coaching seasons later to address the changes in the landscape regarding supportive female coaching provisions. Thematic analysis was adopted to inform the development of emergent themes. Results indicate the need to maintain a fight for continued change in the coaching landscape to enhance the knowledge and understanding of individualised journeys to align appropriate supportive mechanisms for female coaches.

Women's Experiences of Embodied Identity Through Active Leisure Joanne Mayoh $^{1}$ and Ian Jones ${ }^{1}$

${ }^{1}$ Bournemouth University, $U K$

Corporeality is gendered, it is therefore imperative that research explores the uniquely gendered ways in which we experience our bodies. Previous research has highlighted the perceived alienation and feelings of powerlessness associated with women's bodily experiences from multiple perspectives, as well as identifying engagement with physical pursuits as a potential pathway for more positive forms of embodiment. This research aims to contribute to the small yet emerging body of literature that explores how engagement in active leisure may provide existential possibilities for empowerment and the wellbeing for women. In this presentation, we combine theoretical discussion and qualitative empirical data from indepth interviews with women who engage in active leisure activities. Theoretically, we draw upon phenomenologically informed dwellingmobility theory to explore examples of how active leisure provides these opportunities for wellbeing through the experiential lifeworld dimensions of embodiment and identity. Our results demonstrate that active leisure provides a pathway for women to have more empowering body experiences by developing an embodied identity that provides a sense of 'I am my body' or 'my body can'. We suggest that forms of active leisure have the potential for women to have empowering body experiences. We propose the most empowering of experiences are those grounded in a sense of embodied identity dwelling-mobility where these senses are felt simultaneously. In adopting this framework, potential pathways to wellbeing through sport for women are uncovered that can help inform policy and practice.

\section{Differences Between Males and Females in Recovery From Sprint Interval Exercise}

Jacky J Forsyth $^{1}$ and Dean Burt ${ }^{1}$

${ }^{1}$ Staffordshire University, $U K$

The purpose of this study was to examine whether there were differences between males and females in energy metabolism following a bout of sprint interval training (SIT). Sixteen males (aged $M=25.0, S D=$ 4.7 years, height $M=1.81, S D=0.07 \mathrm{~m}$, and mass $M=82.7, S D=13.3 \mathrm{~kg}$ ) and 16 eumenorrheic females (aged $M=26.1, S D=5.5$ years, height $M=$ $1.64, S D=0.09 \mathrm{~m}$, and mass $M=72.0, S D=15.4 \mathrm{~kg}$ ), tested in the midluteal phase of their menstrual cycle, completed a SIT protocol, consisting of $4 \times 30$-s Wingate sprints at $0.065 \%$ fat-free mass. Compared with females, males had significantly $(\mathrm{p} \leq 0.05)$ higher post-SIT mean fat oxidation rates $\left(0.10 \mathrm{~g} \cdot \mathrm{min}^{-1}\right.$ and $0.17 \mathrm{~g} \cdot \mathrm{min}^{-1}$, respectively, $\mathrm{F}_{(1,30)}=$ $34.82, p<0.001)$, energy expenditure $(M=1.28, S D=0.26$ versus $\left.M=1.48, S D=0.35 \mathrm{kcal} . \mathrm{min}^{-1}, \mathrm{~F}_{(1,30)}=20.759, p<0.001\right)$, excess postexercise oxygen consumption values $(M=1.91, S D=0.60$ and $M=3.02$, $\left.S D=1.58 \mathrm{~L}, \mathrm{~F}_{(1,30)}=6.882, p<0.014\right)$. The higher metabolic values postSIT for males might be explained by the males having a greater fat-free mass, since, when values were scaled for FFM, differences between the sexes post-SIT were no longer significant. Potentially, since fat oxidation rates and energy expenditure post-SIT were higher in males, compared to females, SIT might be more successful as a fat-loss intervention in men compared with women.

The Effects of Acute Sodium Bicarbonate Supplementation on High-Intensity Intermittent Performance Using an 80 Minute Rugby-Specific Simulation Test in Elite Female Rugby Players

Paula A Fitzpatrick ${ }^{1}$ and Giles D Warrington ${ }^{2}$

${ }^{1}$ Institute of Technology Carlow, Ireland;

${ }^{2}$ University of Limerick, Ireland

Ingestion of alkalising agents, such as sodium bicarbonate (SB), have been shown to enhance muscle buffering capacity, potentially improving performance. The aim of this research was to examine the effects of acute SB supplementation and a placebo (PLA) on high-intensity intermittent rugbyspecific performance, half-time recovery and subsequent second-half performance in elite female rugby union players. Ten elite female rugby union players volunteered to participate in the study. The study used a 
randomised, double-blind, cross-over design in which participants performed an 80-minute simulated rugby union performance test on two separate occasions. Following a habituation session, each subject completed two experimental trials ingesting an acute pre-exercise dose of either $0.3 \mathrm{~g} / \mathrm{kg}$ body mass of SB or a PLA. Heart rate, blood samples, muscle soreness, gastrointestinal (GI) disturbance levels and rate of perceived exertion (RPE) were taken at specific intervals throughout the testing procedure. Acute SB supplementation resulted in significant alkalosis when compared to PLA $(M=7.47, S D=0.03$ vs. $M=7.38, S D=0.02 \mathrm{pH}$ units for SB and PLA pre-exercise, respectively; $p=0.046)$. However, this did not translate into an ergogenic benefit to rugby union-specific performance. A significant relationship was found between mean GI ratings and performance in the SB trial for elite females. A high degree of individual variability was also revealed. Results of this study indicated that acute SB supplementation with $0.3 \mathrm{~g} / \mathrm{kg}$ body mass has the capacity to induce preexercise alkalosis in elite females. However, conflicting findings were recorded in relation to the ergogenic benefit to rugby union-specific performance.

Sprinting and Endurance Training as a Means to Slow the Age-Related Decline for Females in Bone Strength

Jessica Piasecki ${ }^{1}$, Matthew Piasecki ${ }^{2}$, Alex Ireland ${ }^{3}$, Hans Degens ${ }^{3}$, Jamie $\mathrm{McPhee}^{3}$

${ }^{1}$ Nottingham Trent University, $U K$;

${ }^{2}$ MRC/ARUK Centre for Musculoskeletal Ageing Research and National Institute of Health Research, University of Nottingham, UK;

${ }^{3}$ Manchester Metropolitan University UK

Bone mineral density begins to decline for females at 30 years, with the onset of menopause and loss of oestrogen around 50 years, increasing the likelihood of osteoporosis in older age for non-active females. The relationship between prolonged participation in regular sprint or endurance running and bone geometry at the tibia was examined in female athletes, compared with young inactive controls. Ten female sprinters $(M=62.2, S D=7.9$ years $), 37$ female endurance runners $(M=61.6 S D=$ 5.0 years) and 15 female controls $(M=21.3 S D=3.6$ years) were included for analysis. Both athlete groups had an age-graded performance above $65 \%$, demonstrating considerable athletic ability. Peripheral quantitative computed tomography was used to asses bone geometry at the tibia, and dual x-ray absorptiometry was used to assess body composition (fat and lean mass). ANOVA models with no adjustment, showed the tibia of both athlete groups to have a $\sim 20 \%$ expansion compared to controls, identified by the greater periosteal and endocortical circumference $(\mathrm{p}<0.005)$. However, when adjusting for age and lean mass, there were no significant differences between sprinters, endurance runners or controls in the periosteal or endocortical expansion, nor the cortical density, thickness or area of the tibia at the diaphysis or epiphysis. These data demonstrate that long-term sprinting and endurance running, for females, may slow down the expected age-related decline in bone strength of the lower limbs, resulting in bone geometry and strength similar to young healthy adults. Sprint and endurance running may protect females from later life osteoporosis.

For a Few Dollars More - The Representation of Elite Women's Cricket in Australia

Keith D Parry ${ }^{1}$ and Jo Batey ${ }^{1}$

${ }^{1}$ University of Winchester, $U K$

In 2018, Cricket Australia launched \#WATCHME, a powerful new marketing campaign created to promote the Australian Women's Cricket Team and the Women's Big Bash League (WBBL) - the country's elite professional Twenty 20 national cricket competition.
This paper provides an important empirical snapshot and analysis of number of key areas. Namely, the representation of women's cricket and differences between that of the men's game; differences in the financial value placed on both forms of the game by fans; and the significance of the choice of venue for women's cricket matches. A two-phase mixed methodology was adopted for this study, utilising participant observation in conjunction with quantitative survey data. Participant observation was conducted at four WBBL matches, and an online survey was sent out via the club's email distribution list, yielding 308 responses. The gender breakdown was 159 females (52\%) and 149 males (48\%). The modal age was 18-24 years of age. The findings revealed that there were differences in the presentation of WBBL games compared to those of the male Big Bash League. WBBL games were also observed to have a more family-friendly atmosphere and were less focused on established rivalries and competitiveness. In addition, female fans preferred smaller, suburban venues for these matches. Significantly, it was found that male fans financially value women's sport less than female fans do. These findings indicate that women's sport remains a site of contestation with ongoing struggles over whose version of sport truly matters.

They Were Like, 'Wow, These Girls Can Play Golf!': Women's Experiences of Competing Against Men in a Professional Golf Tournament

\author{
Ali Bowes ${ }^{1}$ and Niamh Kitching ${ }^{2}$ \\ ${ }^{I}$ Nottingham Trent University, UK; \\ ${ }^{2}$ Mary Immaculate College, Ireland
}

The culture of golf is widely regarded as male-dominated, despite women's interest in playing the sport. Typically, women's participation has not been welcomed nor taken seriously, and golf culture has normalised exclusionary and discriminatory practices towards women where females struggle for equality of access, participation, employment and decision making in golf. Due to restrictions on women's involvement, ladies' sections were formed in golf clubs and, subsequently, gender-segregated golf governing bodies were developed independently from the men's game. In May 2018, the Men's European Tour invited five professional female players to compete in its innovative Golf Sixes event in England, against 27 professional male players. This paper draws upon interview data with those five players on their experiences of competing against men, which was thematically analysed. Initial findings shed light on the complexities of sex and gender in a sporting domain, with the women themselves perpetuating understandings on the 'naturalness' of sex differences. Data also indicates a hierarchical power relationship between men and women's golf. Despite this, the women were also found to be advocates for equality, positioning themselves as spokeswomen for professional women's golf.

\section{Female Athletes' Voices: Transgender Guidelines and Single-Sex Sport}

Cathy Devine ${ }^{1}$

${ }^{I}$ Independent researcher

UK and International Olympic Committee guidelines for transgender inclusion in sport are under review. Most sports are 'gender affected' and activities 'of a competitive nature in circumstances ... in which the physical strength, stamina or physique of average persons of one sex would put them at a disadvantage compared to average persons of the other sex as competitors in events involving the activity'. Transwomen can be lawfully excluded from single-sex activities 'if it is necessary to do so to secure in relation to the activity - (a) fair competition, or (b) the safety of competitors'. Feminist political theorists argue girls and women have equal moral 
standing in the political and social structures of society, including sport. As such, their voices should carry equal democratic weight. The voices of elite female athletes are rarely heard in the political and scientific discourse in relation to transgender inclusion in sport, and this paper therefore explores the views of 15 female Olympic athletes. The main findings are: (1) unanimous agreement that both female and transgender athletes should be treated fairly, and (2) unanimous agreement that national governing bodies of sport (NGBs) should revisit the rules for transgender inclusion and be much more transparent regarding the scientific evidence on which they are based. NGBs must uphold the legal requirement for 'fair competition' and 'safety of competitors'. Female athletes are stakeholders, and their views should be sought. NGB rules for the inclusion of transwomen in female sport should be based on robust scientific evidence which is transparent to athletes.

\section{Exploring Women's Leadership in China's Sport National Governing Bodies}

Han Zheng ${ }^{1}$, Aaron Smith ${ }^{1}$, and Emily Hayday ${ }^{1}$

${ }^{1}$ Loughborough University, $U K$

Although the topic of women's leadership has been more widely mentioned in the past decade, in most areas women are still seriously underrepresented at the leadership or decision-making level; while sport, as a long-term male-dominated industry, is no exception. When people turn their attention to China, a country that occupies an important position in world sports, they find that the research on female leadership is very limited. In the past decade, there have been few in-depth studies on Chinese women's leadership in the sports industry. Numerical data indicates that the underrepresentation of women's leadership is still very serious in China. In Chinese enterprises, women occupy only $16 \%$ of the leadership, while in scientific and technological research institutions, women occupy $5.6 \%$ of the leadership. According to the data collected by the authors at present, the average proportion of female occupational leadership in China's national governing bodies (NGBs) is less than $20 \%$. The purpose of this research is to explore the current situation of women leadership in sport governing bodies in China in order to fill the research gap. In this research, a multi-level framework proposed by Leberman and Burton in 2017 was applied. By using both questionnaires and interviews, data on sport NGBs in China were collected. In addition to barriers being classified into three levels, the inner connections between each level will also be explored. Finally, the degree of representation of women's leadership will be explored, while some targeted options will be put forward on how to support women's representation in leadership.

On the Need for Raising the Profile of Elite Women Sport - Bahrain Hussa K Al Khalifa ${ }^{1}$

${ }^{1}$ Loughborough University, UK

A study on the condition of women football in Bahrain reveals athlete retainment at the elite level as a major and long-standing issue. Three main reasons account for this: weak youth systems, high dropout rates and a lack of incentives. Findings indicate that increased co-operation and policy congruence between the Football Association and educational institutions is needed to strengthen youth engagement with football. Social stigma and lack of familial support can be mitigated through media campaigns and outreach programmes to promote awareness on the health benefits of exercise. Beyond this, the aim should be to raise the profile of women sports at par with their male counterparts, especially at the elite level, in order to encourage mass participation from below. This will create positive female role models in sport (which is currently lacking) and increase trophy/ medal-achieving potential for the country. Financial incentives in the form of fixed salaries will prioritise training sessions for athletes who often have to balance other jobs and/or school. As it stands, zero percent of female national team players in any sport receive an income for their efforts, except for bonuses for winning and pocket money during team travels for camps and competitions. Non-financial incentives, such as job or scholarship provision, will also encourage long-term commitment by athletes and yield encouragement by their families. Raising the profile of elite women in sport encourages female athletes to get in the game younger (stronger youth systems), stay in it longer (lower dropout rates) and commit harder (longer retainment).

\section{Perceptions of the Opportunities and Barriers Facing Females When Undertaking Sport-Related Study and Employment}

Jacky J Forsyth $^{1}$, Alison Bambridge ${ }^{1}$, Lyn Duval ${ }^{1}$, and Jennifer Jones ${ }^{1}$

${ }^{1}$ Staffordshire University, $U K$

Males outnumber females in sport-related employment, especially in graduate-level jobs, senior roles and in leadership positions. This trend, which has changed little over time, might reflect the sex-stereotyping and marginalisation of females that is specific to sport. The purpose of this study was to investigate perceptions of sport and exercise career opportunities and barriers among females at different career stages, in order to explore why females are under-represented in sport-related educational and employment settings. Three focus groups were formulated, consisting of high-school students $(n=20)$, university students $(n=8)$ and graduates $(n=7)$, all of whom were studying or working in sport and exercise. Data were transcribed and thematically analysed. All groups perceived there to be gender discrimination and gender stereotyping within sport-related employment, but felt that by working harder and pushing through the barriers, they could overcome these discriminations and be successful in a sport-related career. The graduates, however, seemed to be the most apprehensive of the perceived discrimination, which could result in them terminating their career in sport and exercise. Self-confidence and selfbelief were deemed important for success, but issues around multiple-role conflict, networking and homologous reproduction were perceived to be real, external barriers to progression. Efforts to address gender inequality in sport and exercise were felt to be tokenistic. Strategies, such as reducing internal and external barriers, are still required to reduce gender inequality in career and educational settings relating to sport and exercise.

\section{The Gender Equity Toolkit for Generation Z - Facilitating Gender Equality in Sport Leadership}

Vanessa Jones ${ }^{1}$, Gill Renfree ${ }^{1}$, and Beth Burgess ${ }^{1}$

${ }^{1}$ University of Worcester, $U K$

The Gender Equity Toolkit for Generation Z (GETZ) is a Collaborative Partnership Project co-funded by Erasmus + Sport programme involving seven European partners from a mix of universities and national sport organisations. The aim of the GETZ project is to facilitate gender equality in sport leadership by educating and empowering Generation $\mathrm{Z}$ by providing access to a Massive Open Online Course (MOOC) designed to address issues around gender equality and equal opportunities in sport participation and sport leadership. Technology plays an integral role in the way in which Generation Z communicate, socialise and learn, and has been embraced to engage participants in a range of interactive learning tasks, activities and materials aimed at developing their knowledge, understanding and critical thinking. The MOOC is made up of six individual units each with a specific focus on the issues surrounding the under-representation of females in the participation, leadership and management in sport. An initial research study has been conducted to establish perceptions and attitudes of GZ via year-1 University students from four research institutions involved in the project, and has informed the content of the Units. This flexible educational resource is aimed at 
both males and females - equality between women and men is a fundamental principle of the European Union; equality is enshrined in Article 23 of the Charter of Fundamental Rights of the European Union; and, more specifically, since the inclusion of an EU Sport Competence in the Lisbon Treaty (2009), the issue of gender equality can be addressed within the context of sport.

\section{Women's Barriers to Becoming and Being a Strength and Conditioning Coach}

Jaime Guinan ${ }^{1}$, Gavin Thomas ${ }^{1}$, and Gyozo Molnar ${ }^{1}$

${ }^{1}$ University of Worcester, $U K$

Strength and conditioning (S\&C) coaches work with athletes to improve athletic prowess and prevent injuries. The number of people who seek S\&C positions has increased. The UK Strength and Conditioning Association reported in 2018 that there are 750 members who are accredited coaches. However, only seven (!) percent of those are women. Clearly, female coaches are almost completely absent in $\mathrm{S} \& \mathrm{C}$, especially at the elite-level. To explore women's perceptions and attitudes towards becoming an S\&C coach in predominantly male-dominated sports and to identify specific reasons for women's marginalisation in the sector, we carried out an exploratory study using semi-structured interviews with 15 female S\&C coaches $(M=29.7, S D=6.0$ years old $)$, with a minimum of two years' experience of coaching males and females $(M=9.2, S D=6.4$ years $)$. Participants have worked in the UK $(n=10)$, America $(n=3)$ and Australia $(n=2)$. Interviews were transcribed verbatim, analysed using thematic coding and key themes identified. Participants noted three main challenges, which included: organisational politics, impression management of gender identity and work-life balance. All of these acted as barriers to reaching higher leadership positions or to secure S\&C employment in other sports. A coping mechanism that emerged to deal with the above challenges was the use of humour, specifically banter to create buy-in. The S\&C profession is extensively influenced by sociocultural forces that promote hegemonic masculinity. Specific education programmes to support women in $\mathrm{S} \& \mathrm{C}$ is recommended for the industry to reduce the gender gap.

Factors influencing the Quality of the Coach-Athlete Relationship During the Specialisation Phase in Female Football

Rachael Brient ${ }^{1}$ and Scott Bradley ${ }^{1}$

${ }^{I}$ University of Northampton, Northampton, UK

The Coach-Athlete Relationship (CAR) is a dynamic and dyadic relationship formed between athlete and coach that is supportive of enhanced levels of coaching effectiveness, athlete wellbeing and sport performance. However, a paucity of research exists that has explored coach and athlete perceptions of effective CARs across developmental stages in female sport. Following ethical approval, f female footballers $(n=18)$ and highperformance football coaches $(n=6)$ in specialisation phase age groups (U12, U14, U16), on the FA female talent pathway, completed the coach or athlete version of the coach-athlete relationship questionnaire (CART-Q), and qualitative open-ended questions exploring coach-athlete perceptions of effective CARs. CART-Q scores were directly compared across specialisation phase age groups; responses to open-ended questions were thematically analysed using a constant comparison method and presented as direct textual quotations. The findings indicated: positive trends (complimentarity; co-orientation), stable trend (commitment), and unstable trend (closeness) for CART-Q scores across age groups. Significantly, closeness scores (U16) were lower in comparison with all other age groups. Furthermore, qualitative responses identified that perceptions of CAR supportive conditions varied across age-groups: establishing 'trust' and an 'open dialogue' emerged as common themes for all age groups; 'feeling listened to' emerged as a common theme in the U16 age group only.
Findings suggest that gender and athlete developmental-stage represent influential criteria determining perceived CAR effectiveness in sport. It is recommended that future research is grounded in the context of female sport, and that applied practitioners fully consider the influence of developmental and psychological differences.

\section{'The Faster and Noisier, the Better': Photographic Representation of Empowerment Through Body Performance of an Australian Female Surf Lifesaver}

Kate Russell ${ }^{1}$

${ }^{1}$ University of East Anglia, UK

Media portrayals of female athletes often represent a (hetero)sexualised, trivialised and marginalised account of their sporting participation. It is the juxtaposition between social expectations of femininity placed upon female athletes that can create tension with the physical demands of sport choices, in which a strong, powerful and muscular body shape is demanded. Often, women in sport are seen in passive poses, emphasising femininity. Younger athletes may view these images as representations of what it is to be a 'legitimate' athlete. When female athletes get to choose how an image is constructed, the authentic nature of what an athlete is was valued highly by younger athletes. Forty-one Australian elite athletes took part in a study to explore photographic representations of what it means to be an elite athlete. Athletes chose the location, attire and activity that best represented to them what being an athlete was for a younger audience, and athletes were interviewed about their choices. Maddie is one of these athletes and is a competitive surf lifesaver. Her photos and interview data revealed that representing a strong, physical and empowered body was key to her image as a positive role model for what girls could hope to achieve in the highly masculinised world of competitive lifesaving. Younger athletes, however, felt her images of positive body performances were 'inauthentic' and 'showing off', in opposition to ideals of true representations of a surf lifesaver. Conclusions are drawn in regard to negotiating tensions between body performances, stylised versions of sport and engaging younger athletes.

\section{The Seemingly Innocuous: Sexism and the Sport Psychologist}

Aura Goldman ${ }^{1}$ and Misia Gervis ${ }^{2}$

${ }^{1}$ University of Hertfordshire, UK;

${ }^{2}$ Brunel University, UK

Though sexism has been recognised as problematic in sport, its impact on sport psychologists has not yet been investigated. The authors present a commentary on sexism in the context of sport psychology. To date, this is an issue that has not been discussed in relation to current practices of sport psychologists. Critical aspects of sexism are explored and considered through the practitioner lens to consider the impact it has on the profession. Particular attention is given to the female practitioner experience of working within the 'macho' culture of elite sport. The intention of our commentary is to begin the conversation among sport psychologists with a view to challenge and change the status quo, drawing from preliminary focus group data.

\section{Posters}

The Kinematics of Breasts Implanted With a Reduced Mass Implant: A Preliminary Report

Melissa Jones ${ }^{1}$, Michelle Norris ${ }^{1}$, Chris Mills ${ }^{1}$, Tim Blackmore ${ }^{1}$, and Joanna Wakefield-Scurr ${ }^{1}$

\section{${ }^{1}$ University of Portsmouth, UK}

Breast implants may increase breast skin tension and interact with surrounding tissues to alter breast position and motion during dynamic activity. Reducing implant mass and changing implant location 
(submuscular/subglandular) may also affect breast kinematics and the subsequent load on breast structures. This preliminary report aims to describe the kinematics of breasts augmented with reduced mass implants during standing, walking and running, compared to natural breasts, and to provide insight into how implant location (submuscular/subglandular) alters breast kinematics. At 12 to 15 months post-surgery, two breast augmentation participants (32AA pre-surgery, anatomical submuscular $255 \mathrm{cc} \mathrm{B}^{-L i t e}{ }^{\circledR}$ reduced mass implant, and 32A pre-surgery, anatomical subglandular $285 \mathrm{cc}$ B-Lite ${ }^{\circledR}$ reduced mass implant) and two natural breasted participants of similar breast size and anthropometrics were recruited (Natural 1 and 2). Nipple and torso positional data were recorded using electromagnetic sensors during standing, walking and running. Nipple kinematics relative to the torso were calculated. The B-Lite ${ }^{\circledR}$ participants both displayed greater nipple projection and elevation during standing and a 50\% reduction in nipple acceleration during walking, when compared to their natural counterparts. During running, the B-Lite ${ }^{\circledR}$ subglandular participant displayed decreased nipple kinematics, compared to their natural counterpart, and lower nipple kinematics compared to the B-Lite ${ }^{\circledR}$ submuscular participant during walking and running. A combination of implant location (subglandular) and reduced mass minimised nipple kinematics during running. Reducing nipple kinematics during dynamic activity may decrease the loading on breast structures, helping to decrease ptosis and recovery time, and increase procedure longevity.

\section{The Effects of the Menstrual Cycle on Body Composition Using Ultrasound}

Sarah Hillier ${ }^{1}$, Rebecca Cook $^{1}$, Kristine Staulen ${ }^{1}$, Tais Van Ondheusden ${ }^{1}$, and Cristina Pangua Irigaray ${ }^{1}$

${ }^{1}$ Oxford Brookes University, UK

Women often report weight changes during different phases of the menstrual cycle; however, evidence remains inconclusive in determining if these changes are specifically in relation to fat mass. The aim of this study was to assess body composition during the three phases of the menstrual cycle using the novel ultrasound measurement technique using the IOC Medical Commission protocol. Thirty naturally cycling females were recruited to participate in this study. Participants were tested during three phases of the menstrual cycle (menstrual, follicular and luteal). The follicular phase was identified by detecting the luteinising hormone surge using ovulation sticks. Each visit involved basic anthropometric measurements (height and weight) and body composition assessments using the standardised ultrasound procedures. The ultrasound images captured at the eight body sites were analysed using computer software (FAT, Software, rotosport.com) to measure subcutaneous adipose tissue thickness within the area of interest. Sixteen naturally cycling females (age: $M$ $=32.3, S D=8.0$ years; body mass index: $M=25.1, S D=3.6 \mathrm{~kg} / \mathrm{m}^{2}$ ) completed all measurements. Statistical analysis displayed no significant differences in subcutaneous adipose tissue thickness across all eight sites between the three menstrual cycle phases. This investigation has expanded the limited research, suggesting that menstrual cycle phase has no influence on body composition assessments. This indicates that women can take part in body composition assessments at any time during the menstrual cycle, although further evidence is required to confirm due to small sample size.

\section{Women's Decisions to Exercise in Pregnancy: Negotiating Conflicting Identities}

Jenny Hendley ${ }^{1}$

${ }^{1}$ University of Brighton, $U K$

Despite the recognised benefits of regular physical exercise during pregnancy, only a minority of women meet current pregnancy exercise recommendations, and there is limited understanding of women's decision-making regarding exercise in pregnancy. This constructivist grounded theory study aimed to develop a theoretical insight into the factors that influence women's decisions and how they process the influences and multiple alternatives they encounter. The constructivist approach built inductively from the experiences of 10 pregnant women who exercised regularly, supplemented by insights from fitness experts and extant literature. Longitudinal, audio-recorded, semi-structured interviews occurred twice during each pregnancy and six to eight weeks postnatal. The resultant substantive theory conceptualises pregnancy as a transitional period during which women's self-identity is modified. Decisions regarding exercise were influenced by a complex interplay of contextual factors that simultaneously encouraged exercise and rest. This consequently triggered a degree of identity conflict between two domains of their self-identity: 'the exercising self' and 'the pregnant self'. The women reacted to the challenges to their identity through the process of self-identity regulation. Through this process, they gradually reconstructed their self-identity to accommodate their pregnant self while contemplating possible future selves against various self and social normative standards. The substantive theory underscores the significance of self-identity in steering the decision making process. The findings provide insight into how women might be better supported to make informed and assured decisions regarding lifestyle choices. Drawing on the findings, the author has recently launched a website designed to provide information for women and professionals who advise them: www.womenexerciseforlife.co.uk

\section{Combined Cardiopulmonary Exercise Testing and Uterine Artery Doppler Analysis at 14 and 24 Weeks' Gestation}

Dylan McGagh ${ }^{1}$, Ralph Smith, Henry Boardman, Sarah French, William Bradlow, Sally Collins, Christos Ioannou, Raffaele Napolitano; Yvonne Kenworthy, Wilby Williamson, Peter Cox, Eduardo Lima, Paul Leeson, and Lucy Mackillop ${ }^{2}$

${ }^{1}$ University of Oxford, $U K$;

\section{${ }^{2}$ John Radcliffe Hospital, Oxford, UK}

There is an absence of evidence regarding the impact of strenuous intensity exercise on pregnancy. Cardiopulmonary exercise testing (CPET) has been used among pregnant women and allows the implementation of individually graded exercise intervals. Uterine artery doppler velocimetry is a measure of uteroplacental perfusion. This pilot study investigates the effects of increased exercise intensity on uteroplacental blood flow in pregnant women at two gestational time points. Ten healthy pregnant women (aged $M=35, S D=4.3$ years, body mass index $M=24.3, S D=3.3 \mathrm{~kg} . \mathrm{m}^{2}$ ) were prospectively recruited; six were nulliparous. At each visit (14 and 24 weeks), a foetal ultrasound scan was performed. Three bouts of four minutes of fixed resistance workload at $40 \%, 60 \%$ and $80 \%$ of maximum peak $\mathrm{VO}_{2}$ was performed, with uterine artery assessment performed at each intensity and a blood test at the start and finish. Combined CPET and doppler studies were successfully completed in seven participants. During the CPET test, the peak workload achieved was similar at both visits. There was a significant increase in lactate before and after maximal exercise in both visits. Mean uterine artery doppler indices did not significantly change with exercise. Most notably, the pulsatility index after $80 \%$ of maximal exertion did not significantly change versus baseline measurement at either visit. All participants delivered healthy live neonates at term. We successfully demonstrated combined uterine doppler analysis, and CPET studies is well-tolerated in pregnant women and that graded exercise did not adversely impact on uterine artery doppler values in this small cohort. 
Exercise Professionals' Confidence in Engaging Women to be Physically Active During Pregnancy

Stacey Draper ${ }^{1}$, Marlize de Vivo ${ }^{1}$, and Hayley Mills ${ }^{1}$

${ }^{I}$ Canterbury Christ Church University, UK

Physical activity (PA) levels significantly decline during pregnancy, even for women who were previously active. The responsibility for providing this guidance is often passed between exercise and health professionals, leaving pregnant women confused, consequently, favouring inactivity. This study aimed to determine: (1) what experiences exercise professionals (EP) have in engaging pregnant women to be physically active; (2) perceived confidence around engaging pregnant women in PA; and (3) whether different experiences were associated with different levels of confidence in engaging women in PA during pregnancy. Ninety-eight EP completed an online survey assessing demographics, experiences and confidence (items adapted from the Self-Confidence in Patient Education for Health Promotion questionnaire. Independent sample t-tests were used to compare mean scores between groups. Nearly a quarter of participants $(22.4 \%)$ admitted to turning away pregnant women on at least one occasion. EP who had children; were female; those who read about or researched the topic; those who were aware of the current recommendations; those who participated in continuing professional development activities involving the topic; and those who held a Level 3 qualification, all had significantly $(p<0.05)$ higher total confidence scores. The findings suggest that fitness qualifications (below Level 3) are not equipping EP with the: (1) knowledge to advise pregnant women regarding the risks/benefits; (2) skills to convey appropriate PA information to pregnant women; and (3) confidence to actively engage pregnant women. Both exercise and health professionals have a role to play in normalising PA behaviour during pregnancy through consistent and clear messages.

Exercise Interventions for Preventing and Treating Low Bone Density in the Wrist and Forearm Among Women: A Systematic Review and Meta-Analysis

Opeyemi O Babatunde ${ }^{1}$, Amy L Bourton ${ }^{2}$, Karen Hind ${ }^{3}$, Zoe Paskins ${ }^{1,4}$ and Jacky $\mathrm{J}$ Forsyth ${ }^{5}$

${ }^{1}$ Keele University, $U K$;

${ }^{2}$ University of Nottingham; $U K{ }^{3}$ Durham University, $U K$;

${ }^{4}$ Haywood Academic Rheumatology Centre, Staffordshire \& Stoke-onTrent Partnership Trust;

${ }^{5}$ Staffordshire University, $U K$

The purpose of this study was to examine the effectiveness of exercises for improving forearm bone mass among pre- and postmenopausal women. Databases (MEDLINE, EMBASE, CINAHL, AMED, Web of Science, and Cochrane CENTRAL) were searched, from their inception until December 2018. Eligibility criteria included women undertaking upper-limb exercise interventions ( $\geq 12$ weeks) to improve bone mass. Screening of titles/abstracts/full-texts and data extraction were undertaken independently by pairs of reviewers. Included studies were quality appraised using Cochrane risk of bias tool. Exercise interventions were classified into: 'resistance training' of high or low intensity (RTHI/ RTLI), or 'impact'. 2,180 unique citations were screened, 165 studies reviewed for full-text eligibility, and 24 studies were included in the review, of which 21 provided suitable data for meta-analysis. Quality ranged from 'low' to 'unclear' risk of bias. Exercise generally led to increases in forearm bone mass $(\mathrm{SMD}=1.27, \mathrm{Z}$-value $=4.10, p<$ $0.001)$. RTHI $(\mathrm{SMD}=1.00, \mathrm{Z}$-value $=3.11, p=0.002)$, and RTLI $(\mathrm{SMD}=2.36, \mathrm{Z}$-value $=2.33, p<0.001)$ led to moderate increases in forearm bone mass. Improvements due to impact exercises (SMD = $1.12, \mathrm{Z}$-value $=0.92, \quad p=0.36$ ) were not statistically significant.
Subgroup analysis, based on menopausal status, showed that postmenopausal women were more likely to benefit from upper-body exercise interventions $(\mathrm{SMD}=1.64, \mathrm{Z}$-value $=4.46, p<0.001)$ than were premenopausal women. In summary, there was moderate-quality evidence that exercise is effective for improving forearm bone mass, especially in postmenopausal women.

\section{Female Ultra-Endurance Athletes: An Exploration of Their Pain Experiences}

Britta Maria Sorensen ${ }^{1}$, Carla Meijen ${ }^{1}$, and Stacy Winter ${ }^{1}$

'St Mary's University, UK

Experiencing pain and discomfort is an accepted part of ultra-endurance participation. Athletes have different perceptions of how they experience, tolerate, and deal with pain in ultra-endurance competition, although less is known about the experiences of female ultra-athletes. Therefore, this study adopted a biopsychosocial approach to explore females experiences and management of pain during training and competition for ultra-endurance events. Interpretive Phenomenological Analysis (IPA) guidelines informed semi-structured interviews with 13 participants (aged $M=44.08 ; S D=$ 5.40 years). Ultra-endurance sports included triathlon, trail-running, and open-water swimming. Participants competed at recreational, professional, and amateur age-group national levels. Topics explored how participants perceived and tolerated different types of pain, how learning from experience helped future performance, and which factors influenced or shaped their pain management. Themes, identified through IPA, represented the interpretations of the pain experiences and management. Key influencing factors of their ability to 'ignore' pain during a race included maintaining positive mind-sets, perceived high tolerance levels, and a determined resilience to keep going. Life experiences, reframing pain, and learning from races developed a greater self-awareness for controlling their mind, body, and the situation. A practical implication for athletes, coaches, and psychologists to consider, is the emergent juxtaposition between ignoring pain during a race, general denial of pain, and balanced awareness between potential self-harm and preservation. Female ultra-athlete mental wellbeing was wrapped up in their need for continuing training despite 'niggles'. Longitudinal mixed-method research is recommended to monitor any fluctuating factors that could influence pain management during a competitive season.

\section{Narrative Review: The Mental Health and Wellbeing of Rugby Players}

Shakiba Oftadeh-Moghadam ${ }^{1}$ and Paul Gorczynski ${ }^{1}$

${ }^{1}$ University of Portsmouth, UK

Rugby is a physically and psychologically demanding contact sport. Due to the high rates of injury, previous research has traditionally focused on physical injuries, rather than symptoms and disorders of mental illness in rugby. Consequently, the aim of this review was to synthesise evidence regarding the mental and wellbeing of rugby players. A narrative review was conducted. The studies were required to meet the following inclusion criteria: (1) study must be written in English; (2) participants must be rugby players; (3) the article must investigate a topic/s assessing the mental health and/or wellbeing of rugby players. Studies were excluded from the review if the study was undertaken with a heterogenous sport sample. After a systematic search, 11 studies were included in the review. Players that were forced to retire were more likely to report symptoms of distress in comparison to those who retired voluntarily. Athletes who reported a history of five or more concussions were more likely to report symptoms of mental health disorders, in comparison to former athletes reporting no concussions. Only one study included rugby players who identified as women. Rugby players, regardless of gender, may not seek support for 
mental health problems, for reasons such as stigma and a lack of mental health literacy. Moreover, the mental health and wellbeing of women rugby players is neglected and therefore requires more rigorous, highquality intervention-based studies. Future research should prioritise this to inform interventions aimed at increasing the mental health literacy of rugby players that identify as women.

\section{Differences in Men and Women Volunteers Within a Parkrun Environment}

Gill Renfree ${ }^{1}$ and Julia West ${ }^{1}$

${ }^{1}$ University of Worcester, $U K$

Women were found to volunteer more frequently and for more hours than men. In society, women tend to volunteer more than men; however, in sport, they are less likely to volunteer than men. This study investigates potential differences in motivation and commitment between men and women volunteering in local parkrun communities. After receiving ethical approval from the academic institution and parkrun Research Board, 92 volunteers (male $n=45$; female $n=47$ ) completed an online survey comprising demographic information (age, employment status, gender), the Organizational Commitment Questionnaire and the volunteer motivation questionnaire. The majority of participants were in employment $(n=76)$ and only 12 participants were retired. Data were analysed using a series of Mann Whitey- $U$ tests, and results showed that women reported significantly higher levels of parkrun pride $(M=5.93, S D=1.26, p=0.02, z=-2.33)$, egoistic motivation $(M=3.09, S D=0.89, p=0.02, z=-2.22)$ and leisure motives $(M=2.81$, $S D=.87, p=.003, z=-3.01)$ than men $(M=5.43, S D=1.26 ; M=2.64, S D=$ 0.79 and $M=2.26, S D=0.72$ respectively). Women reported higher levels of motivation and commitment to parkrun events than men reflecting a greater desire for social approval through being valued within the parkrun environment. This aligns with the parkrun philosophy which creates a welcoming and highly valued approach for all its volunteers.

\section{This Woman Does! Exploring Working Women's Relationship With} Physical Activity and Exercise

Holly Collison $^{1}$ and Ksenija Kuzmina ${ }^{1}$

\section{${ }^{1}$ Loughborough University London, UK}

Part funded by Sport England, this research directly feeds into the significant gap that exists in current knowledge focused on women in the workforce and the social, employment and domestic challenges working women experience. It is through a sociological lens that we explored the realities of teachers and office workers by constructing ten contextual case studies to visualise and explore their complex and layered realities. The methodological design took a layered approach which included: (1) Interviews; (2) Fitbit; (3) Photo voice; (4) Observations; (5) Self-persona; and (6) Video captured sense-making. Self-quantification data was used as a 'learning technology' where personal data was collected by and about individuals using a mediating technology. Research focused on the tension between concepts of 'perceived activity levels' vs 'actual activity level', as well as 'mechanical objectivity' where activity levels are measured through numbers and 'situated objectivity'. By combining and sympathetically applying a mixed-method approach, this research employed a unique and highly original and interdisciplinary design. Some preliminary outcomes included: social networks within the workplace are highly influential on motivation and regular participation in physical activity. Women had a desire to be more active but felt the workplace and workloads was generally not supportive or conducive to exercising once the working day had finished. Self-perception ranged from feelings of laziness and selfcriticism to being task masters in their working roles with little space to prioritise their sense of wellbeing. In a comparative frame, internal cultures enabled collective activity around workspace and time.

The Dose-Effects of Caffeine on Lower Body Maximal Strength, Muscular Endurance, and Rating of Perceived Exertion in Strength Trained Females

Louise Jones ${ }^{1}$ and Andrew Hulton ${ }^{1}$

${ }^{1}$ University of Surrey, $U K$

Caffeine is well established as an ergogenic aid for endurance, highintensity and team sport performance. It is yet to be determined whether different doses of caffeine can improve parameters of resistance training, especially in females. Therefore, the current study aimed to investigate the effect of pre-exercise caffeine ingestion on lower body maximal strength and muscular endurance in strength-trained females, and to identify any dose-response. In a double-blind, randomised, counterbalanced design, 10 strength-trained females (age: $M=22.7$, $S D=3.6$ years; BMI: $M=23.2, S D=2.9 \mathrm{~kg} / \mathrm{m}^{2}$ ), using hormonal contraception, consumed either 3 or $6 \mathrm{mg} . \mathrm{kg} \mathrm{BM}-1$ of caffeine or placebo (PLA) at least three days apart. Thirty minutes following supplementation, participants performed a one-repetition maximum (1RM) leg press and repetitions to failure (RF) at $60 \%$ of their 1RM. Rating of perceived exertion (RPE) was recorded every five repetitions, and total volume lifted during the RF test was calculated. Repeated measures ANOVA indicated no significant differences in 1RM, RPE, number of repetitions or total volume achieved between conditions. There was a trend for number of repetitions to improve by $19 \%$ with $3 \mathrm{mg} . \mathrm{kg}$ BM-1 caffeine $(M=43.4, S D=18.1 ; p=0.069)$ and by $26 \%$ with $6 \mathrm{mg}$. kg BM-1 caffeine $(M=46.2, S D=25.5 ; p=0.082)$ compared to PLA $(36.6 \pm$ 14.6). These findings suggest that caffeine doses of 3 and 6 mg.kg BM-1 do not improve maximal strength or RPE in strength-trained females. There was a trend for caffeine to improve muscular endurance and total volume completed. The small sample size, among other limitations, may explain the lack of significance. 